\title{
INCREASED RETENTION OF A PARTIAL DENTURE MAXILLARY OBTURATOR USING DENTAL IMPLANT
}

\author{
N. Yunus ${ }^{1}$, Z.A.A. Rahman ${ }^{2}$. Increased retention of a \\ partial denture maxillary obturator using dental implant. \\ Annal Dent Univ Malaya 2000; 7: 51-55.
}

\section{ABSTRACT}

Tissue-integrated oral implants have opened-up a new perspective in oral rehabilitation of tumour patients who had undergone surgery. The present case demonstrated a simple approach to rehabilitate a patient who had subtotal maxillectomy using dental implant. The use of an implant in combination with a natural abutment tooth was shown to improve the retention and stability of the obturator. Magnetic attachment and telescopic restoration were the retainers of choice and they provided good aesthetic result.

Key word: Implant, maxillectomy, retention, esthetics, obturator, magnetic attachment, telescopic restoration.

\section{INTRODUCTION}

The application of osseointegrated implants in dentistry has provided a means of restoration for compromised maxillectomy patients. The use of implants to support and retain maxillary obturator prostheses had been promising (1-4). These cases reported dramatic success in reconstruction of totally edentulous maxillectomy patients with multiple implants.

In dentate maxillectomy patients, the support, retention and stability of the prosthesis can be achieved by making use of the residual teeth, the available tissue undercuts and the support area within the defect (5). The supplemental use of osseointegrated implants in these patients may further increase the longevity of the remaining dentition especially those teeth adjacent to the surgical defect (6). This article describes the rehabilitation of a patient with a maxillary defect due to tumour resection using implant. The use of implant in combination with a natural abutment tooth to provide additional retention to the partial denture maxillary obturator is described.

\section{PATIENT HISTORY}

The patient, a 16 year-old Chinese female student presented with a lump at the junction of left soft and hard palate area in November 1995. Incisional biopsy was done and the histopathological examination reported as low-grade mucoepidermoid carcinoma. Computerised Tomography Scan had shown extension of lesion into left maxillary sinus and left posterior choanae and invasion of left pterygoid plate. A surgical procedure involving subtotal maxillectomy of left maxilla with
Case Report

N. Yunus ${ }^{1}$ and Z.A.A. Rahman

'Department of Prosthetic Dentistry, and
'Department of Oral \& Maxillofacial Surgery
Faculty of Dentistry
University of Malaya
50603 Kuala Lumpur
Malaysia
Corresponding author - N. Yunus

removal of most of right hard and soft palate was done in December 1995 (Figure 1). The lost tissue was replaced with an acrylic obturator (Figure 2). In February 1998, she was planned for insertion of osseointegrated implant to improve the stability and retention of her prosthesis. After detailed exploration of the oral cavity under general anaesthesia, it was found that the only suitable area for the implant was at the premaxilla adjacent to the apical area of left central and lateral incisors. A $13 \mathrm{~mm}$. implant (Branemark, Nobel Biocare, Sweden) was then inserted at this area

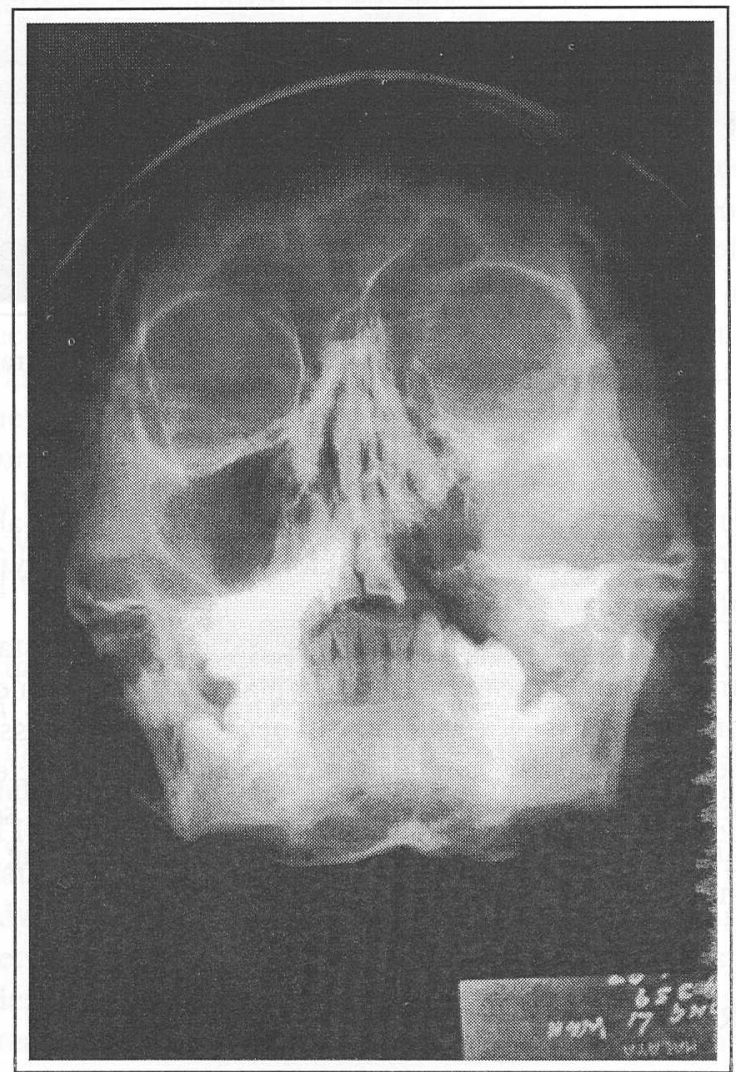

Figure 1: A radiograph showing the extent of bone loss after maxillary resection. 


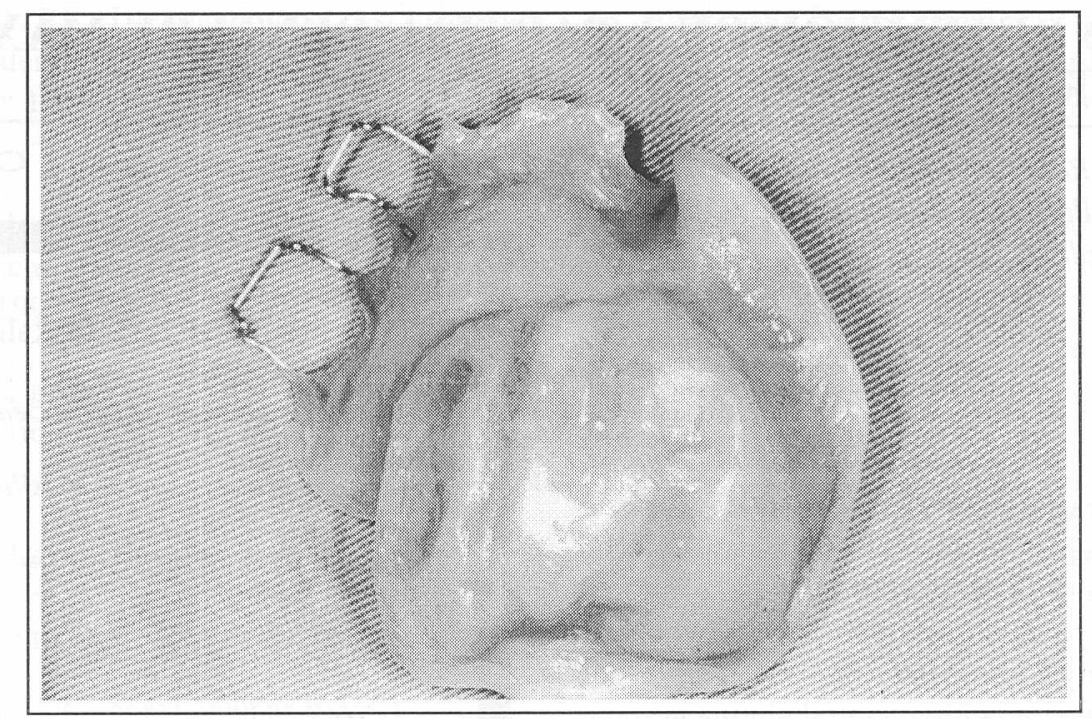

Figure 2: The fitting surface of the acrylic obturator with adequate extension into defect area. However, note the lack of anterior clasping as this was not presentable to the patient.

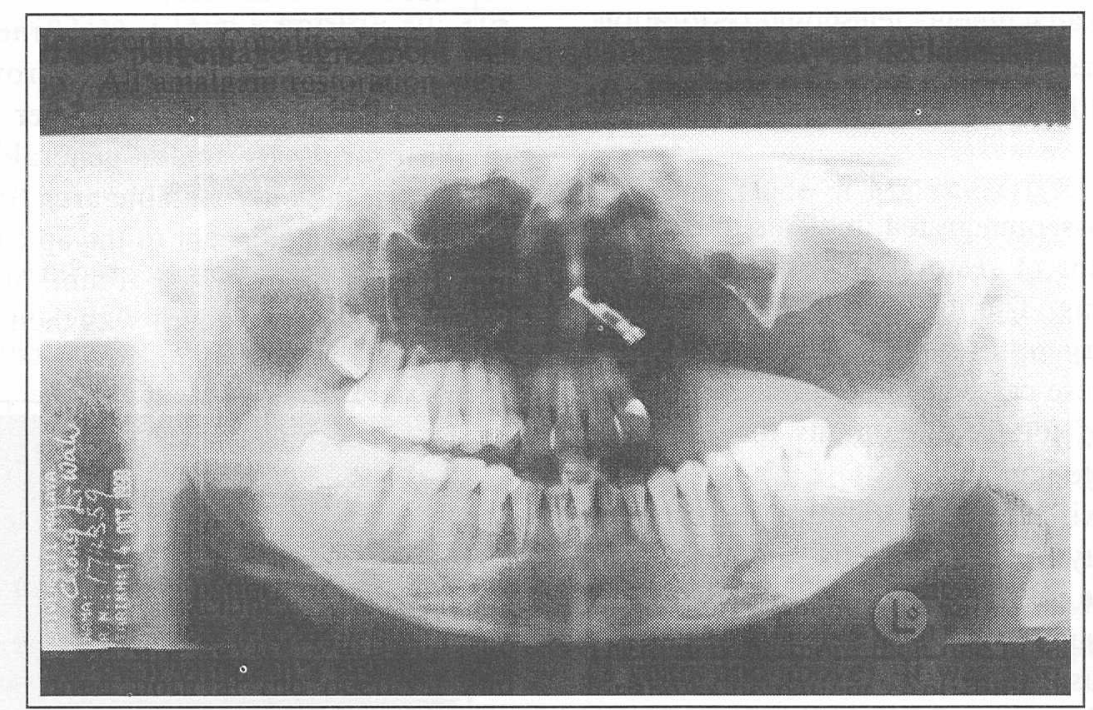

Figure 3: Orthopantomograph showing the position of the implant.

(Figure 3). The right zygomatic area was not suitable because of the presence of thick and dense overlying soft tissue.

After 8 months of osseointegration, second-stage surgery for exposure of the implant was performed. Some soft tissue was excised because of the tissue overgrowth and a $7 \mathrm{~mm}$ healing abutment was connected. Her existing obturator was adjusted and relined with soft lining material. Two weeks after soft tissue healing, a $5 \mathrm{~mm}$ standard abutment was connected.

\section{PROSTHETIC PROCEDURE}

A maxillary preliminary impression was made using irreversible hydrocolloid impression material in a stock tray, with some modification of the defect with impression compound. The study model was surveyed, the path of insertion selected and the framework designed. The same basic principle of obturator Class II design for partially edentulous patient (5) was followed. However, instead of having a conventional cast clasp as the retainer on the anterior terminal abutment, a conical double-crown telescopic restoration was preferred (7). Since the upper left lateral incisor was heavily filled and required crowning it was prepared with a deep chamfer margin. An impression of the preparation was made in polyether using a custom-made tray. The purpose was to guide the construction of the inner crown. A $6^{\circ}$ taper was created along the crown length with the direction of taper to be within the path of insertion of the framework. Retention was achieved 


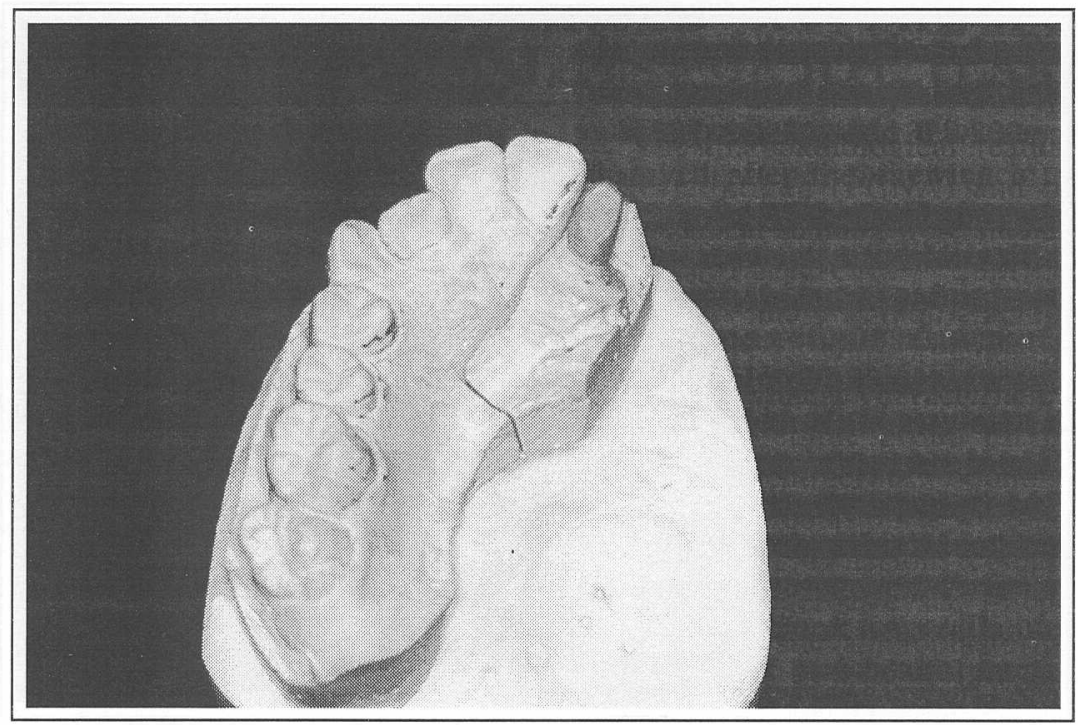

Figure 4: The inner crown casting of $\mid 2$ on the working model before polishing. The path of insertion of the denture frameowrk has to coincide with the taper angle of the crown.

in this portion through contact surfaces of the inner and outer crowns. The taper was created using a special bur on a milling machine. The coping was casted in Ag$\mathrm{Au}-\mathrm{Pd}$ alloy (Figure 4) and verified for fit in the mouth. Final milling and polishing would be done at the time of the construction of the outer crown.

A master impression was made in a custom-made tray prepared with an extension of the defect area. A tapered impression coping was screwed onto the implant abutment and the inner crown seated on the prepared left lateral incisor. The defect area and the denture border were moulded in impression compound and the final impression was made in polyether material. The inner crown was picked-up in the impression. The tapered impression coping was removed from the implant abutment and connected to an abutment replica before being repositioned on to the impression. The master cast was formed with the inner crown and the implant abutment replica recorded in the correct relation to the remaining teeth and the defect. Because of the angulation of the implant to the occlusal plane, as verified from the master cast, it was decided to incorporate a magnet-keeper attachment system as the retainer.

The outer crown for the lateral incisor and the cobalt-chromium framework were constructed and checked for fit intraorally. They were soldered together in the correct relationship before the prosthesis was processed in acrylic resin.

Jaw relationship was recorded and maxillary teeth try-in was done in the usual manner.

At the delivery stage the inner crown was cemented to the abutment using zinc polycarboxylate cement. A stainless steel magnetic keeper was screwed onto the implant abutment (Figure 5). The magnet was attached to the fitting surface of the obturator by direct intraoral pick-up using a chair-side cold-cured resin (Figure
6). The occlusion and the fit were checked and refined at the follow-up stage.

\section{DISCUSSION}

One of the difficulties in rehabilitating maxillectomy patients using implants is the lack of alveolar bone qualitatively and quantitatively for implant placement. Various techniques of bone grafting had been developed so as to improve the bone volume. Gary et al (8) augmented bone graft in defect and non-defect site for implant anchorage and reported that the obturator had improved in function. However bone grafting procedure would require great surgical skill. It will also involve additional surgery to the patient, as simultaneous implant insertion at the time of bone grafting is not recommended (9). In this case presentation, the patient was not prepared to undergo this procedure. Therefore the alternative was to place the implant at the available bone which was at the remaining premaxilla adjacent to the defect area. This segment was considered as a key site for implant anchorage because of the quality of the bone (10).

The use of magnetic attachment in angulated implant situation solved the different paths of insertion problem in the prosthesis. Other advantages of using magnets were that the laboratory procedure was less complicated and it transmitted less lateral forces to the abutment (11). The combination use of an implant and a terminal natural abutment with telescopic crown was advantageous as it helped to redistribute the forces between them. As the angulation of the implant was not ideal, the use of another abutment adjacent to the defect could avoid overloading. Parr (12) advocated splinting a few abutments near the defect as additional protection. Telescopic crown also gave good esthetic as it 


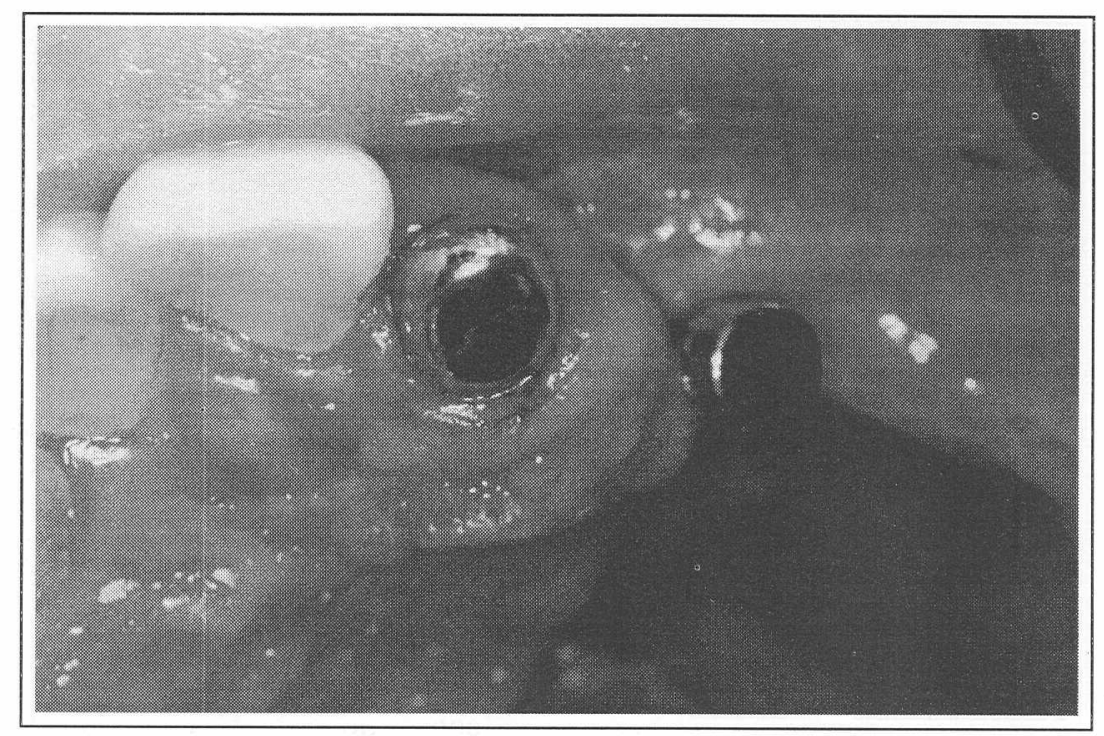

Figure 5: Inner crown after cementation on the lateral incisor and the keeper after being screwed onto the implant abutment.

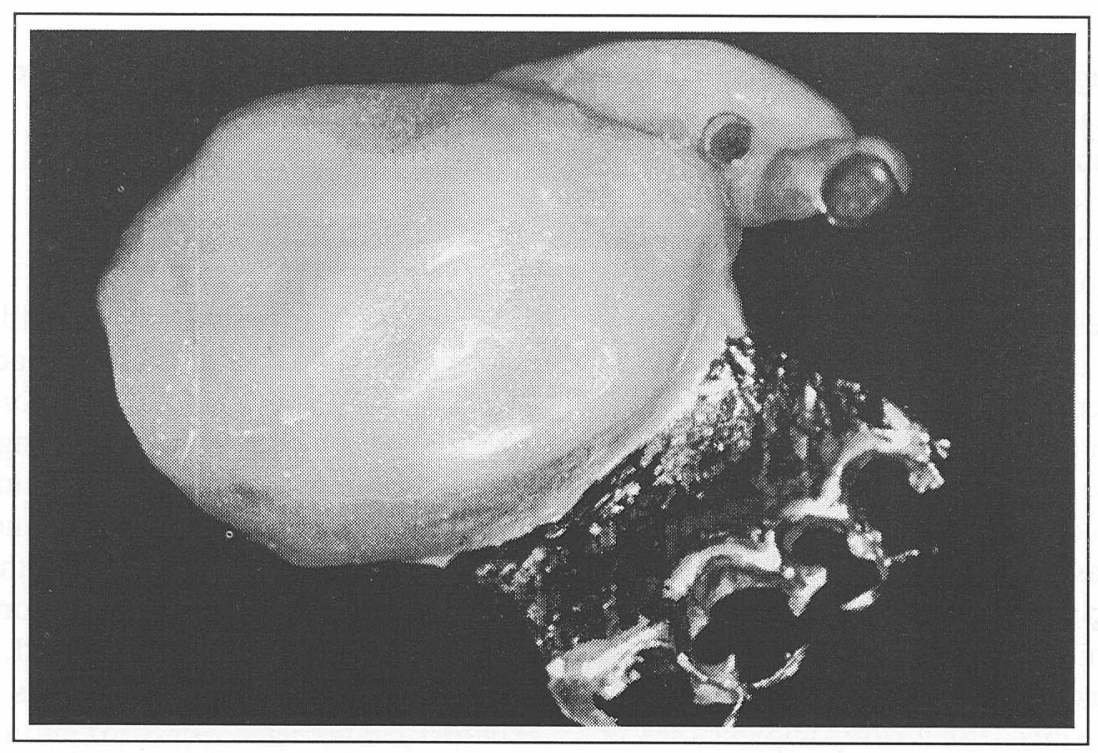

Figure 6: The fitting surface of the prosthesis showing the extension of the obturator bulb and the position of the magnet after chairside curing.

eliminated the use of anterior cast clasp for retention. The large surface contact area of parallel walls in the double crowns provided good retention. Moreover the degree of retention could be planned to suit different situations by modifying the design. Another advantage of telescopic retainer over clasps was the more axial transfer of occlusal load that produced less rotational torque upon the abutment tooth (13). This was important especially in obturator prostheses where the amount of torque generated by the movement could be enormous.

The patient reported that the obturator now functioned satisfactorily because of the added retention gained from the magnet and telescopic crown. She was also satisfied with the appearance of her teeth (Figure 7). At the present time, the patient is being closely monitored for her overall oral hygiene and the implant stability. The long-term success of this treatment however cannot be predicted at this time.

\section{CONCLUSION}

The patient treatment demonstrated the added value of an integrated implant in augmenting retention in a maxillary obturator. A non-rigid magnetic retainer in combination with a conical double-crown telescopic restoration provided increased retention and stability of an otherwise conventional partial denture maxillary obturator. 


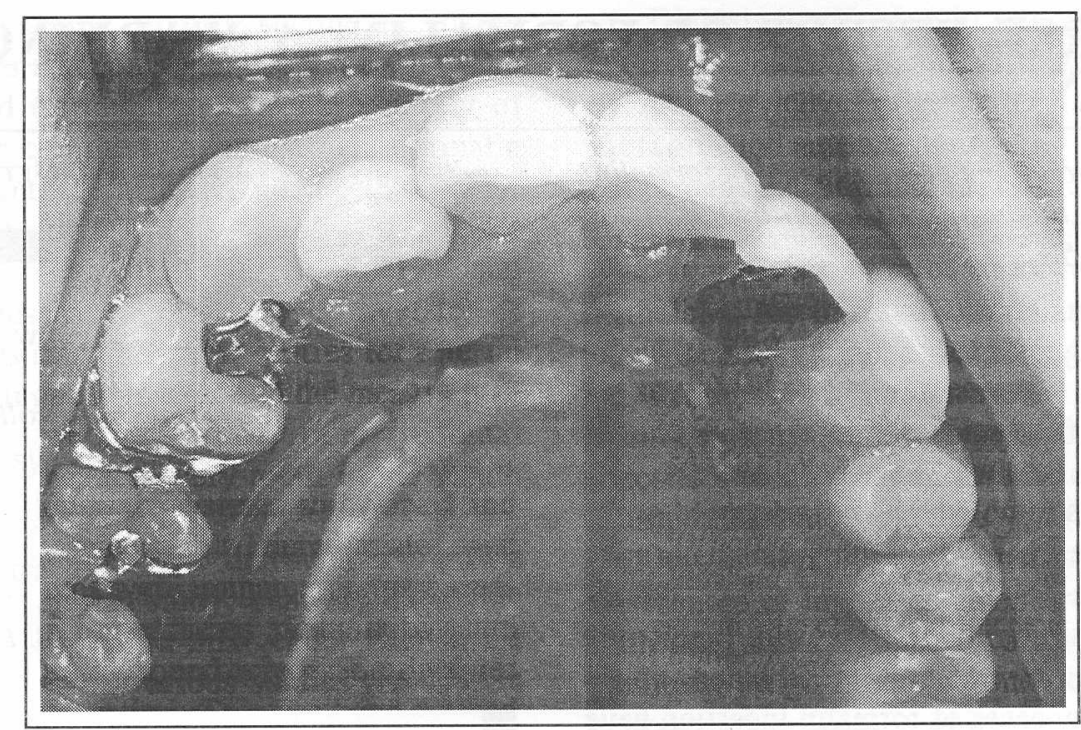

Figure 7: The use of magnetic attachment and telescopic retianer anteriorly avoids wire clasping which can be unaesthetic.

\section{REFERENCES}

1. Desjardins RP. Obturator prosthesis design for acquired maxillary defects. J. Prosthet. Dent 1978; 39: 424-34.

2. Langer A. Telescope retainers for removable partial dentures. J. Prosthet. Dent 1981; 45: 37-43.

3. Shiba A. The conical double-crown telescopic removable periodontic prosthesis. Ishiyaku EuroAmerica, Inc. 1993; 1: 4-9.

4. Parr CR. Swing-lock design considerations for obturator frameworks. J. Prosthet. Dent 1995; 74: $503-11$.

5. Roumanas ED, Nishimura RD, Davis BK, et al. Clinical evaluation of implants retaining edentulous maxillary obturator prostheses. J. Prosthet. Dent 1997; 77: 184-9.

6. Gary JJ, Donovan M, Garner FT, et al. Rehabilitation with calvarial bone grafts and osseointegrated implants after partial maxillary resection: A clinical report. J. Prosthet. Dent 1992; 67: 743-6.

7. Block MS, Guerra LR, Kent JN, et al. Hemimaxillectomy prosthesis stabilization with hydroxyapatite-coated implants: A case report. Int. J. Oral Maxillofac. Implants 1987; 2: 111-3.
8. Niimi A, Ueda M, Kaneda T. Maxillary obturator supported by osseointegrated implants placed in irradiated bone: Report of cases. J. Oral Maxillofac Surg 1993; 51: 804-9.

9. Mentag PJ, Kosinski TF. Increased retention of a maxillary obturator prosthesis using osteointegrated intramobile cylinder dental implants: A clinical report. J. Prosthet. Dent 1988; 60: 411-4.

10. Izzo SR, Berger JR, Joseph AC, et al. Reconstruction after total maxillectomy using an implant-retained prosthesis: A case report. Int. J. Oral \& Maxillofacial Implants 1994; 9: 593-5.

11. Parel SM, Branemark PI, Jansson T. Osseointegration in maxillofacial prosthetics. Part I: Intraoral applications. J. prosthet. Dent 1986; 55: 490-3.

12. Schliephake H, Neukam FW, Schmelzeisen R, et al. Long-term results of endosteal implants used for restoration of oral function after oncologic surgery. Int. J. Oral Maxillofac. Surg. 1999; 28: 260-5.

13. Pezzoli M, Highton R, Caputo A, et la. Magnetizable abutment crowns for distal-extension removable partial dentures. J. Prosthet. Dent 1986; 55: 475-9. 